\title{
Fully Affine Invariant Methods for Cross-Session Registration of Calcium Imaging Data
}

\author{
Chunyue Li, Xiaofeng Yang, Ya Ke, and Wing-Ho Yung
}

https://doi.org/10.1523/ENEURO.0054-20.2020

School of Biomedical Sciences and Gerald Choa Neuroscience Centre, Faculty of Medicine, The Chinese University of Hong Kong, Shatin, Hong Kong 999077

\begin{abstract}
In vivo two-photon microscopy permits simultaneous recording of the activity of the same neuronal population across multiple sessions in days or weeks, which is crucial for addressing many fundamental questions of neuroscience. The field-of-view (FOV) alignment is a necessary step for identifying the same neurons across multiple imaging sessions. Accurate FOV alignment becomes challenging in the situations of image blurring, insufficient common neurons, or uneven background brightness. The existing methods largely fail to align FOV pairs in these situations. The fully affine invariant approach has been applied in computer vision to register real scene images with different backgrounds. However, its performance in calcium imaging data is unknown. We explored the feasibility of using the fully affine invariant approach to align calcium FOV images across multiple sessions by examining the performance of five methods. Further, we compared their performance with common feature-based methods as well as some classical methods with or without adaptive contrast enhancement. Using cellular resolution calcium imaging data recorded from two areas of the mouse motor cortex over weeks, we show that all fully affine invariant methods provide more accurate FOV alignment results than other methods in general and in the case of a few common neurons identified, uneven background brightness or image blurring. This study demonstrated the feasibility and reliability of the fully affine invariant methods in cross-session FOV alignment. These methods could be useful for neuroscience research, especially on questions that involve experience-dependent plasticity spanning over days or weeks.
\end{abstract}

Key words: cross-session field-of-view alignment; fully affine invariant approach; two-photon calcium microscopy

\section{Significance Statement}

Field-of-view (FOV) alignment is challenging when neurons collected in two sessions are not one-to-one mapped or calcium data are recorded under different imaging parameters and brain states. For the first time, we explored the feasibility of using the fully affine invariant methods to align calcium FOV images across multiple sessions and compared their performance with many conventional methods and their variations. We demonstrate that fully affine invariant methods outperform other conventional methods and are robust under unfavorable conditions. Our work is important for studies on experience-dependent processes, such as learning and memory. Moreover, although fully affine invariant methods are conducted on two-photon calcium imaging data, these methods should be promising in FOV alignment of one-photon or widefield fluorescence microscopy.

Received February 5, 2020; accepted July 16, 2020; First published July 24, 2020.

The authors declare no competing financial interests.
Author contributions: C.L., Y.K., and W.-H.Y. designed research; C.L. and X.Y. performed research; C.L. analyzed data; C.L., Y.K., and W.-H.Y. wrote the paper. 


\section{Introduction}

In vivo two-photon calcium imaging on rodents is a crucial technique for studying many fundamental questions in the field of neuroscience such as visual processing and motor control (Han et al., 2019; Hwang et al., 2019; Stringer et al., 2019). This technique is particularly useful for studying brain mechanisms of learning and memory as it allows researchers to record experience-dependent changes of neurons over extended periods of time in awake behaving animals (Grewe et al., 2017; Pakan et al., 2018; Namboodiri et al., 2019; Wagner et al., 2019).

To chronically follow neurodynamics, the same group of neurons must be reliably registered across multiple sessions (or days). The field-of-view (FOV) alignment is a necessary step for cell registration (Kaifosh et al., 2014; Sheintuch et al., 2017; Giovannucci et al., 2019). However, several factors could induce uncertainty and potential errors in the FOV alignment, rendering it a rather challenging step for cell registration. First, manual head-fixing in each recording session can easily lead to viewing angle change in addition to $\mathrm{X}-\mathrm{Y}$ plane translation and rotation, making a one-to-one mapping of neural identity not necessarily attainable. Second, for long-term recording, the quality of the microscopic image may decrease because of photobleaching, phototoxicity, and brain state change (Lichtman and Conchello, 2005), necessitating the use of different acquisition parameters, which could result in different background intensities and signal-to-noise ratios across sessions. Lastly, for rodents, the common problem of dural regrowth increases with the number of sessions, which reduces optical transparency and leads to image blurring (Heo et al., 2016).

In the past, many efficient methods have been used to register the calcium FOV across multiple sessions. These include classical intensity-based methods, such as TurboReg (Thévenaz et al., 1998), Lucas-Kanade (LK; Baker and Matthews, 2004), and enhanced correlation coefficient (ECC; Evangelidis and Psarakis, 2008). There are also approaches like MOCO (Dubbs et al., 2016) and non-rigid NoRMCorre (Pnevmatikakis and Giovannucci, 2017) used by the popular CalmAn toolbox (Giovannucci et al., 2019). Recently, feature-based approach, such as scale-invariant feature transform (SIFT; Lowe, 2004), Speeded Up Robust Features (SURF; Bay et al., 2008), Accelerated-KAZE (AKAZE; Alcantarilla and Solutions, 2011), Binary Robust Invariant Scalable Keypoints (BRISK; Leutenegger et al., 2011), and Oriented FAST and Rotated BRIEF (ORB; Rublee et al., 2011) have also been used in microscopic image alignment (Stanciu et al., 2010; Ûnay and Stanciu, 2018; Chen et al., 2019). However, because of different reasons, these techniques could fail in the situations of image

Acknowledgements: We thank Dr. Danny C W. Chan for the involvement in the collection of raw images.

Correspondence should be addressed to Ya Ke at yake@cuhk.edu.hk or Wing-Ho Yung at whyung@cuhk.edu.hk.

https://doi.org/10.1523/ENEURO.0054-20.2020 Copyright @ 2020 Li et al.

This is an open-access article distributed under the terms of the Creative Commons Attribution 4.0 International license, which permits unrestricted use, distribution and reproduction in any medium provided that the original work is properly attributed. blurring, insufficient common neurons or uneven background brightness. Given the limitations, a new approach in FOV alignment that could achieve more robust results is much warranted.

ASIFT (Yu and Morel, 2009, 2011) is a fully affine invariant method. It simulates all possible affine distortions caused by the viewing angle changes and then applying the SIFT method to compare keypoints detected from all the simulated images. ASIFT can acquire more keypoints than SIFT even in the situation of negligible or moderate camera view angle change (Yu and Morel, 2011), which means that ASIFT could be applicable for calcium FOV alignment. Moreover, the principles of ASIFT, i.e., matching keypoints detected in both original images as well as affine simulations, can be extended to other similar invariant matching methods, such as SURF, AKAZE, ORB, and BRISK, making them potential solutions for calcium activity FOV alignment. However, the performance of ASIFT and extended fully affine feature-based methods [AffineSURF (ASURF), Affine-AKAZE (AAKAZE), Affine-BRISK (ABRISK), and Affine-ORB (AORB)] on calcium imaging data is unknown.

In this study, we investigated the performance of ASIFT, ASURF, AAKAZE, ABRISK, and AORB on crosssession FOV alignment of calcium imaging data. By using L1-norm, we decreased their unreliability caused by the random sample consensus (RANSAC; Fischler and Bolles, 1981). Further, we compared their performance with general feature-based methods, i.e., SIFT, SURF, AKAZE, BRISK, and ORB, widely used methods, i.e., LK, ECC, MOCO, TurboReg, and NoRMCorre, as well as these widely used methods combined with a contrast-limited adaptive histogram equalization (CLAHE; Reza, 2004). For convenience, the four groups of methods are named as the fully affine invariant group, feature-based group, the conventional group, and CLAHE-based conventional group, respectively. We found that the fully affine invariant group is superior to other methods even in the situation of image blurring, insufficient common neurons, and uneven background brightness. As far as we know, this is the first study that demonstrated the feasibility of the fully affine invariant approach in cross-session FOV alignment of calcium imaging data.

\section{Materials and Methods}

\section{Data collection}

Calcium imaging data were collected from layer $2 / 3$ in the rostral forelimb area (RFA) and caudal forelimb area (CFA) of the primary motor cortex with a custom-built in vivo two-photon microscope while a male C57 mouse learned a two-dimensional (2D) lever reaching task (Fig. 1A). GCaMP6f was injected into the RFA and the CFA to express GCaMP6f in all neuron types. Two weeks after virus injection, the skull located above the recording areas was removed, and the brain surface was covered with a glass coverslip. Behavioral training and two-photon imaging began two weeks after the window surgery. The mouse received one session ( $\sim 100$ trials) per day and 17 sessions in total. Each FOV had a size of $512 \times 512$ pixels and the acquisition frequency was $15 \mathrm{~Hz}$. The 
A

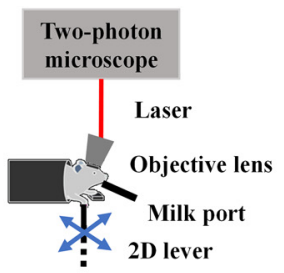

B

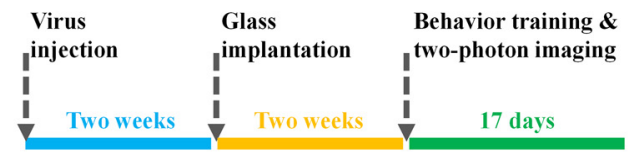

C

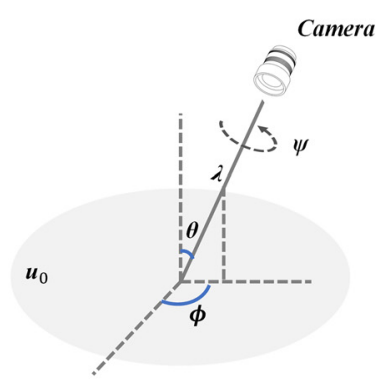

D

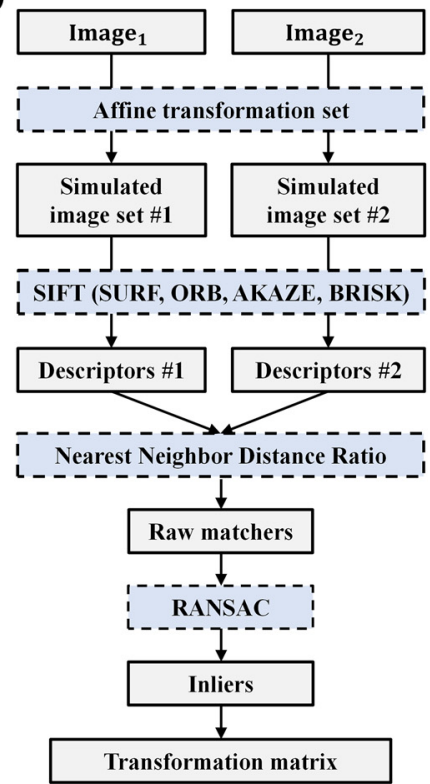

E

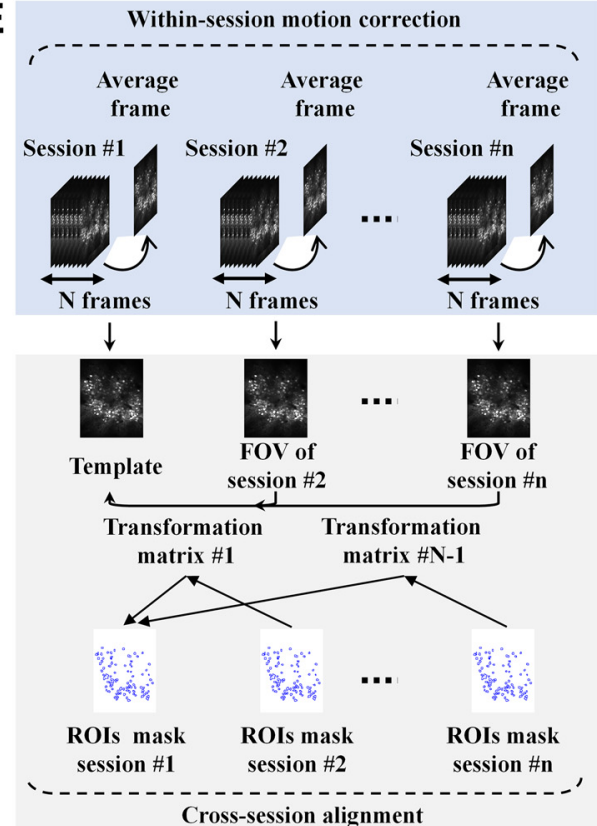

Figure 1. Illustration of experimental design and the proposed FOV alignment approach. $\boldsymbol{A}$, In vivo set up for two-photon imaging data collection. B, Experimental procedure. The GCaMP6f was injected into the RFA and CFA of the layer 2/3 motor cortex. Two weeks later, a cranial window surgery was conducted above the RFA and CFA. Behavioral training and two-photon imaging recording began two weeks after the window surgery. The mouse received one session per day and 17 sessions in total. $\boldsymbol{C}$, Geometric interpretation of the affine decomposition. $\lambda$ and $\psi$ are the zoom factor and the rotation angle of the camera around the optical axis respectively. $\phi$ and $\theta$ corresponds to the longitude and latitude angles of the optical axis. $\boldsymbol{u}_{0}$ represents the frontal view of the flat object. $\boldsymbol{D}$, Generic phases of the ASIFT method. Image $\mathbf{1}_{\mathbf{1}}$ and Image $_{\mathbf{2}}$ were individually transformed by simulating a large set of affine distortions caused by the change of longitude $\phi$ and latitude $\theta$. Then, SIFT was used to detect and describe the keypoints on every simulated image. NNDR was used to match the keypoints. RANSAC was used to exclude outliers from initial matches. The remaining inliers were used to estimate the transformation matrix. SIFT was replaced by SURF, AKAZE, BRISK, and ORB to achieve ASURF, AAKAZE, ABRISK, and AORB. $\boldsymbol{E}$, Outline of the FOV alignment procedure. TurboReg was used to process within-session motion artifacts. The motion-corrected imaging session was averaged and normalized to get the corresponding FOV image. The FOV image of the first session was used as the template, and FOV images of all other sessions were aligned to it. The alignment was achieved by fully affine invariant methods (ASIFT, ASURF, AAKAZE, ABRISK, AORB), the feature-based methods (SIFT, SURF, AKAZE, BRISK, ORB), the conventional methods (LK, ECC, MOCO, TurboReg, NoRMCorre), and the CLAHE-based conventional methods (LK-CLAHE, ECC-CLAHE, MOCO-CLAHE, TurboReg-CLAHE, NoRMCorre-CLAHE).

experimental procedure is summarized in Figure 1B. All animal procedures were performed in accordance with the Chinese University of Hong Kong animal care committee's regulations.

\section{Regions of interest (ROIs) mask}

Neuron detection and FOV alignment are two necessary steps for cell registration. Neuron detection aims to obtain a ROIs mask for each imaging session. The ROls mask includes coordinates or spatial footprints of all active neurons that appeared in one session. FOV alignment seeks to transform the ROls mask from different sessions into one single coordinate system. Cellpose (Stringer et al., 2020) was applied to the mean calcium image of each session to get the corresponding ROls mask. Cellpose can precisely segment neurons of various types and without the needs of model retraining or parameter adjustments. Since dendritic spines can easily be misdetected as neurons, we excluded them by requiring that the $\mathrm{ROI}$ mask of individual neurons should contain at least 60 pixels. Then, each ROls mask was binaried so that the pixel value within a neuron is 255 and the pixel of other places is set as 0 . In this study, the paired raw ROls masks were represented as $\left\{R O / s_{\text {Mask }}^{\text {raw }}{ }_{n}, R O / s_{\text {Mask }}^{\text {raw }}\right.$ template $\}$. Here, the template was defined as the first session; $n$ defined the session index. In addition, the neurons that existed in both template session and each registered session were manually selected and were saved in $\left\{R O / s_{\text {Mask }}{ }_{n}^{\text {common }}, R O l s_{\text {Mask }}\right.$ template $\}$, which was 
used to evaluate the performance of different alignment methods.

\section{ASIFT method}

Generally, a digital image $\boldsymbol{u}$ of a flat physical object $\boldsymbol{u}_{0}$ can be expressed as $\boldsymbol{u}=\boldsymbol{G}_{\mathbf{1}} \boldsymbol{A} \boldsymbol{u}_{\mathbf{0}}$. Here, $\boldsymbol{G}_{\mathbf{1}}$ is a Gaussian kernel modeling the optical blur, and $\boldsymbol{A}$ is a planar protective map. Moreover, if the shape of $\boldsymbol{u}_{\mathbf{0}}$ is locally smooth, the protective map $\boldsymbol{A}$ can be simplified to an affine map. Therefore, the local deformation model is $\boldsymbol{u}(x, y) \rightarrow \boldsymbol{u}(a x+$ by $+e, c x+d y+f)$ in each image region $(x, y)$, where $\boldsymbol{A}=\left[\begin{array}{ll}a & b \\ c & d\end{array}\right]$ represents an affine map and $\left(\begin{array}{l}e \\ f\end{array}\right)$ represents the translation. Further, the affine map $\boldsymbol{A}$ with positive determinant can be decomposed as

$$
A=\lambda\left[\begin{array}{ll}
\cos \psi & -\sin \psi \\
\sin \psi & \cos \psi
\end{array}\right]\left[\begin{array}{ll}
t & 0 \\
0 & 1
\end{array}\right]\left[\begin{array}{ll}
\cos \phi & -\sin \phi \\
\sin \phi & \cos \phi
\end{array}\right],
$$

where $\lambda$ and $\psi$ denote the zoom factor and the rotation angle of camera around optical axis, respectively; $\phi$ and $\theta=\arccos (1 / t)$ represents the longitude and latitude angles of the optical axis, respectively. Figure $1 C$ shows the geometric interpretation of the affine decomposition.

Suppose Image $\boldsymbol{I}_{\mathbf{1}}=\boldsymbol{G}_{1} \boldsymbol{A}_{\mathbf{1}} \boldsymbol{u}_{\mathbf{0}}$ and Image $_{\mathbf{2}}=\boldsymbol{G}_{\mathbf{1}} \boldsymbol{A}_{\mathbf{2}} \boldsymbol{u}_{\mathbf{0}}$ are two digital images of the same object $\boldsymbol{u}_{0}$ taken with different affine map $\boldsymbol{A}_{\mathbf{1}}$ and $\boldsymbol{A}_{\mathbf{2}}$, respectively. To register Image $\boldsymbol{1}_{\mathbf{1}}$ and Image $_{2}$, each of them was individually transformed by simulating a large set of affine distortions caused by the change of longitude $\phi$ and latitude $\theta$. The simulation was achieved by varying $\phi$ with the change of $\theta$, with the step $\Delta \phi=\left(72^{\circ} / t\right), t=(1 / \cos \theta), \psi \in[0, \pi]$, and $\theta \in[-\pi / 2$, $\pi / 2]$. This operation enables ASIFT to be invariant to viewing angle changes. Then, SIFT was used to detect and describe the keypoints on simulated images. The keypoints detected by SIFT are invariant to translation, rotation, and scaling. Therefore, ASIFT becomes a fully invariant method by combining SIFT and the affine simulation.

In this study, SIFT was replaced by SURF, AKAZE, BRISK, and ORB to achieve ASURF, AAKAZE, ABRISK, and AORB. After feature detection and description, the nearest neighbor distance ratio (NNDR) was used to match the keypoints detected in two simulated image sets (Lowe, 2004). The threshold ratio of NNDR was set as 0.75 . Then, outliers were further excluded from initial matches by using RANSAC with 150,000 iterations and 99.9\% confidence. The transformation matrix was estimated using the remaining inliers. Figure $1 D$ presented the procedure of the fully affine invariant approach.

\section{FOV alignment}

FOV alignment included two steps: within-session motion correction and cross-session alignment. The intensity-based method TurboReg was used to process withinsession motion artifacts because calcium imaging data collected within each session have a similar intensity distribution. Specifically, for each session, the average image was taken as the template, and all other calcium frames within this session were registered to the template. Then, the motion-corrected calcium session was averaged and normalized to get the corresponding FOV image. For cross-session alignment, the FOV image of the first session was used as the template, and FOV images of all other sessions were aligned to it. The alignment was achieved by fully affine invariant methods (ASIFT, ASURF, AAKAZE, ABRISK, AORB), the feature-based methods (SIFT, SURF, AKAZE, BRISK, ORB), the conventional methods (LK, ECC, MOCO, TurboReg, NoRMCorre), and the CLAHEbased conventional methods (LK-CLAHE, ECC-CLAHE, MOCO-CLAHE, TurboReg-CLAHE, NoRMCorre-CLAHE). The derived transformation matrix was applied on $R O / s_{\text {Mask }}{ }_{n}^{\text {common }}$, where $n$ defines the session index. The correlation between $R O / s_{\text {Mask }}{ }_{n}^{\text {common }}$ and $R O / s_{\text {Mask }}$ temmolate was used to evaluate the performance of these methods. Specifically, the 2D ROls masks were first reshaped into 1D vectors, then the Pearson's linear correlation coefficient between these vectors was calculated. The ROI masks are binary images containing only 0 or 255 . Therefore, the higher the correlation coefficient, the more similar the ROIs masks. The FOV alignment procedure was summarized in Figure $1 E$.

\section{Reliability improvement of the fully affine invariant group and the feature-based group}

NNDR was used to find initial matches of keypoints for both the fully affine invariant group and the feature-based group. Further, RANSAC was used to exclude outliers from the initial matches. However, in theory, RANSAC cannot ensure to eliminate all outliers and preserve all inliers (Chen et al., 2019). If some important inliers are missed, the registered image will be distorted. Moreover, RANSAC could produce different results each time because of its randomness (Hast et al., 2013). To obtain reliable and reproducible results for both two groups, we repetitively run the NNDR and RANSAC 100 times for each image pair, then choose the transformation matrix which minimizes the L1-norm of the intensity difference of where neuron appears between $\left\{R O / s_{\text {Mask }}^{\text {raw }}, R O{ }_{\text {Mask }}^{\text {raw }}\right.$ template $\}$.

\section{CLAHE for the conventional group}

Uneven background brightness of the FOV image will decrease the performance of the methods in the conventional group. Therefore, CLAHE was used to enhance the contrast of FOV images. Specifically, CLAHE divided an image into multiple non-overlapping blocks. For each block, the histogram was clipped and redistributed to avoid overenhancement. Further, bilinear interpolation was used for adjacent blocks to avoid the appearance of block artifacts. After contrast enhancement, methods in the conventional group were applied on CLAHE adjusted FOV images, and their results were compared with the fully affine invariant group.

\section{Image quality metrics}

A sharpness metrics was used to evaluate image blurriness. Image sharpness is defined as the ratio of high-frequency components above a certain threshold to all pixels in an image (De and Masilamani, 2013). The lower the value, the more blurred the FOV image. The high-frequency threshold was calculated by $M / 1000 . M$ is the 

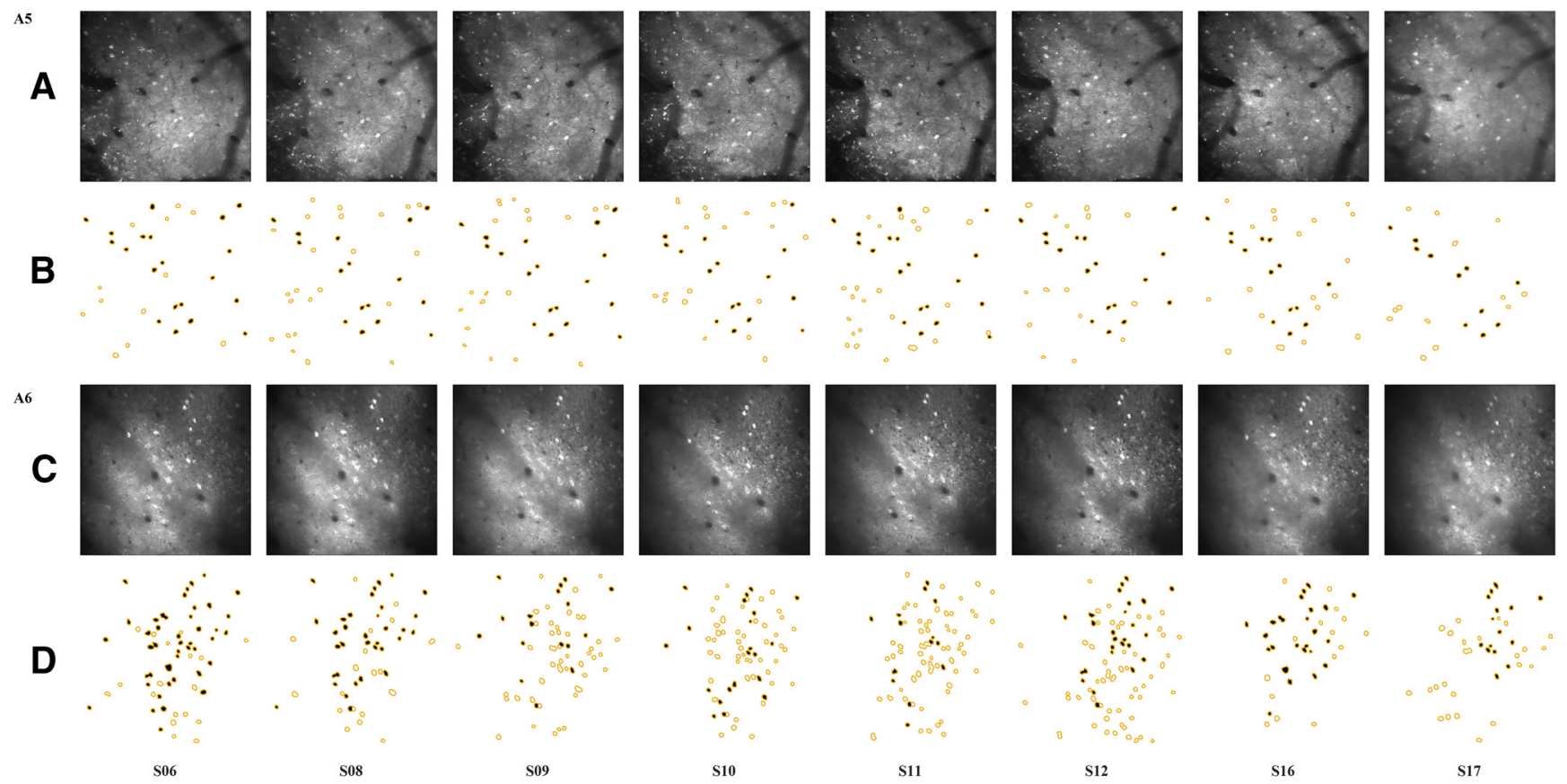

S11

S12

S16

S17
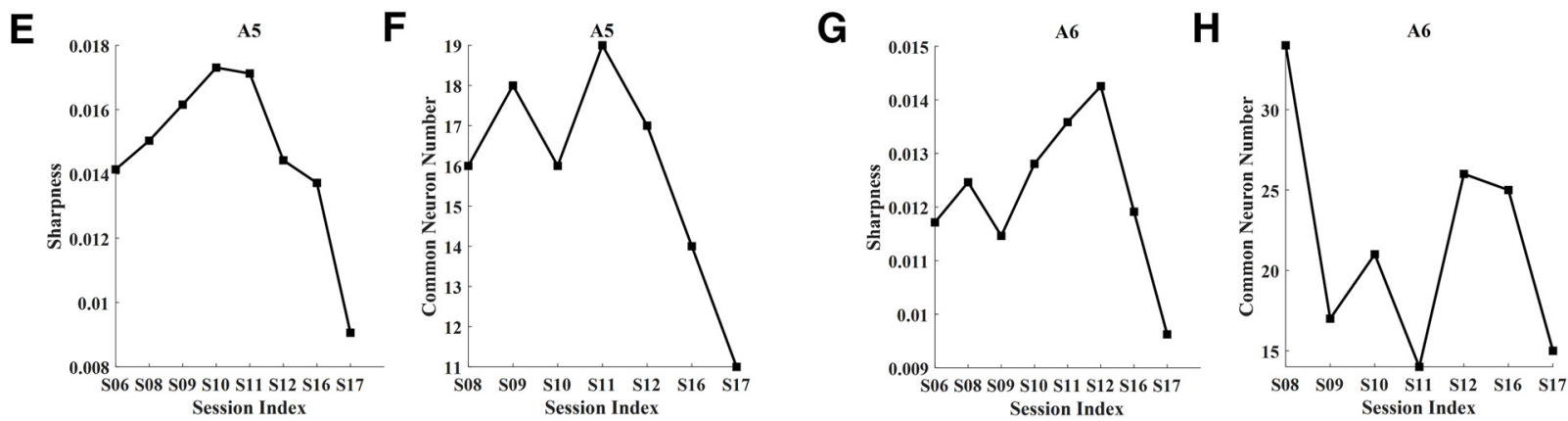

Figure 2. Basic information of the collected FOV images. $\boldsymbol{A}$, The FOV image of each session from RFA, labeled as A5. $\boldsymbol{B}$, The ROls mask of each session from A5. $\boldsymbol{C}$, The FOV image of each session from CFA, labeled as A6. $\boldsymbol{D}$, The ROls mask of each session from A6. The yellow circles represent the detected neurons in each FOV image. The yellow circles filled with black color represent the neurons common to each $R O{ }_{1} s_{\text {Mask }}{ }_{n}^{\text {raw }}$ and $R O / s_{\text {Mask template. }} n$ defines the session index. S is short for session. $\boldsymbol{E}$, The sharpness metrics of each session for A5. The lower the value, the more blurred the FOV image. $\boldsymbol{F}$, Number of neurons common to each $R O / s_{\text {Mask }}{ }_{n}^{\text {raw }}$ and ROls Mask $_{\text {template }}^{r}$ for A5. G, The sharpness metrics of each session for A6. $\boldsymbol{H}$, Number of neurons common to each $R O I s_{\text {Mask }}^{\text {raw }}$ and ROIs Mask template for A6.

maximum value of the centered Fourier spectrum of the FOV template. It has been shown that this particular threshold value gives a fairly accurate sense of image quality (De and Masilamani, 2013).

The other metrics is the number of neurons common to each $R O / s_{\text {Mask }}^{\text {raw }}$ and $R O / s_{\text {Mask template }}^{\text {raw }}$. This metrics measures the content similarity of the FOV image pairs. Usually, the higher the similarity, the more the common neurons.

\section{Codes and computational hardware}

Opencv-contrib-python3.4.2.17 (Bradski, 2000) was used to perform the function of CLAHE, feature-based methods, and fully affine invariant methods. Python package pyStackReg (https://pypi.org/project/pystackreg/) was used for TurboReg. MATLAB 2020a was used to run LK, ECC, and non-rigid NoRMCorre. Codes of LK and
ECC were from the online IAT toolbox (Evangelidis, 2013). Codes of non-rigid NoRMCorre were public online. Fiji was used for MOCO.

All above codes were performed on a Windows 10based laptop equipped with an Intel i7-5500U CPU running at $2.40 \mathrm{GHz}$ and $16 \mathrm{~GB}$ RAM.

\section{Code accessibility}

The code described in the paper is freely available online at https://github.com/chunyueli/FAIMCalcium. The code is available as Extended Data.

\section{Results}

\section{In vivo calcium imaging data}

Calcium imaging data of eight sessions with irregular session-interval collected from two cortical areas RFA 
Table 1. Quantitative comparison between the fully affine invariant group and the feature-based group with respect to area A5

\begin{tabular}{|c|c|c|c|c|c|c|c|c|c|c|c|}
\hline Methods & $\begin{array}{l}\text { Features } \\
\text { in imas } \\
\text { Image }\end{array}$ & $\begin{array}{l}\text { detected } \\
\text { ge pair } \\
\text { Template } \\
\text { (S06) }\end{array}$ & Inliers & $\begin{array}{l}\text { Matched } \\
\text { features }\end{array}$ & $\begin{array}{l}\text { Inlier } \\
\text { ratio }\end{array}$ & Methods & $\begin{array}{l}\text { Features } \\
\text { in ima }\end{array}$ & $\begin{array}{l}\text { detected } \\
\text { ge pair } \\
\text { Template } \\
\text { (S06) }\end{array}$ & Inliers & $\begin{array}{l}\text { Matched } \\
\text { features }\end{array}$ & $\begin{array}{l}\text { Inlier } \\
\text { ratio }\end{array}$ \\
\hline ASIFT & 10636 & 9524 & 95 & 241 & 0.39 & SIFT & 629 & 565 & 10 & 17 & 0.59 \\
\hline ASURF & 20012 & 18698 & 131 & 427 & 0.30 & SURF & 1258 & 1174 & 17 & 42 & 0.40 \\
\hline AAKAZE & 3120 & 2893 & 55 & 89 & 0.62 & AKAZE & 236 & 209 & 11 & 18 & 0.61 \\
\hline \multicolumn{12}{|c|}{ Image pair \#2 of A5 (S09, S06) } \\
\hline ASIFT & 11357 & 9524 & 82 & 195 & 0.42 & SIFT & 690 & 565 & 12 & 22 & 0.55 \\
\hline ASURF & 20398 & 18698 & 98 & 397 & 0.25 & SURF & 1346 & 1174 & 17 & 36 & 0.47 \\
\hline AAKAZE & 3267 & 2893 & 47 & 91 & 0.52 & AKAZE & 269 & 209 & 10 & 16 & 0.63 \\
\hline ABRISK & 9830 & 7667 & 50 & 80 & 0.63 & BRISK & 1169 & 894 & 15 & 26 & 0.58 \\
\hline AORB & 14111 & 13427 & 43 & 96 & 0.45 & ORB & 500 & 500 & 9 & 17 & 0.53 \\
\hline AORB & 14387 & 13427 & 18 & 103 & 0.17 & ORB & 500 & 500 & 4 & 8 & 0.50 \\
\hline \multicolumn{12}{|c|}{ Image pair \#4 of $A 5(S 11, S 06)$} \\
\hline ASIFT & 13833 & 9524 & 61 & 163 & 0.37 & SIFT & 850 & 565 & 9 & 26 & 0.35 \\
\hline ASURF & 22345 & 18698 & 83 & 407 & 0.20 & SURF & 1446 & 1174 & 8 & 32 & 0.25 \\
\hline AAKAZE & 3753 & 2893 & 30 & 76 & 0.39 & AKAZE & 291 & 209 & 7 & 10 & 0.70 \\
\hline ABRISK & 11149 & 7667 & 18 & 44 & 0.41 & BRISK & 1243 & 894 & 11 & 18 & 0.61 \\
\hline AORB & 14514 & 13427 & 20 & 105 & 0.19 & ORB & 500 & 500 & 4 & 5 & 0.80 \\
\hline \multicolumn{12}{|c|}{ Image pair \#5 of $\mathrm{A} 5(\mathrm{~S} 12, \mathrm{~S} 06)$} \\
\hline ASIFT & 10106 & 9524 & 71 & 176 & 0.40 & SIFT & 588 & 565 & 13 & 20 & 0.65 \\
\hline ASURF & 20415 & 18698 & 71 & 404 & 0.18 & SURF & 1293 & 1174 & 7 & 35 & 0.20 \\
\hline AAKAZE & 3239 & 2893 & 47 & 90 & 0.52 & AKAZE & 240 & 209 & 15 & 19 & 0.79 \\
\hline ABRISK & 8477 & 7667 & 29 & 50 & 0.58 & BRISK & 874 & 894 & 6 & 9 & 0.67 \\
\hline ASIFT & 3945 & 9524 & 20 & 69 & 0.29 & SIFT & 202 & 565 & 4 & 5 & 0.80 \\
\hline ASURF & 12914 & 18698 & 20 & 242 & 0.08 & SURF & 649 & 1174 & 6 & 27 & 0.22 \\
\hline AAKAZE & 1802 & 2893 & 13 & 33 & 0.39 & AKAZE & 128 & 209 & 5 & 8 & 0.63 \\
\hline ABRISK & 3092 & 7667 & 12 & 26 & 0.46 & BRISK & 201 & 894 & 4 & 4 & 1.00 \\
\hline AORB & 9454 & 13427 & 13 & 85 & 0.15 & ORB & 498 & 500 & 7 & 14 & 0.50 \\
\hline \multicolumn{12}{|c|}{ Mean values for all image pairs } \\
\hline ASIFT & 10366.14 & 9524 & 55.29 & 161.71 & 0.34 & SIFT & 620.43 & 565 & 8.86 & 17.43 & 0.51 \\
\hline ASURF & 19762.43 & 18698 & 68.57 & 368.71 & 0.19 & SURF & 1247.57 & 1174 & 9.71 & 34.43 & 0.28 \\
\hline AAKAZE & 3163.86 & 2893 & 33.28 & 69.29 & 0.48 & AKAZE & 239.14 & 209 & 8.71 & 14.43 & 0.60 \\
\hline ABRISK & 8498.43 & 7667 & 25 & 48.26 & 0.52 & BRISK & 925.14 & 894 & 8.57 & 13.86 & 0.62 \\
\hline AORB & 13449.29 & 13427 & 28.14 & 104.86 & 0.27 & ORB & 499.71 & 500 & 7.71 & 14.85 & 0.52 \\
\hline
\end{tabular}

and CFA (labeled as A5 and A6, respectively) of a mouse were used in this study. For each imaging area, the FOV image of the first recorded session (labeled as S06) was selected as the template. Figure $2 A, C$ shows the FOV image of each session from A5 and A6. As can be seen, the background brightness of the FOV images were uneven and varied across sessions. The yellow circles in Figure $2 B, D$ represent the detected neurons in ROls Mask $_{n}^{\text {raw }}, n \in($ S06, S08, S09, S10, S11, S12, S16, S17). The yellow circles filled with black color represent the neurons common to each $R O \mathrm{ls}_{\text {Mask }}{ }_{n}^{\text {raw }}$ and $R O \mathrm{Is}_{\text {Mask }}$ raw template $^{\text {. }}$ As can be seen, the common neurons varied across sessions, so there was no one-to-one mapping between the template session and the registered session.

\section{Image quality evaluation}

Figure $2 E, G$ shows the sharpness metrics of each FOV image from A5 and A6. The results indicate that session 17 (S17) has the minimum sharpness value for 
Table 2. Quantitative comparison between the fully affine invariant group and the feature-based group with respect to area A6

\begin{tabular}{|c|c|c|c|c|c|c|c|c|c|c|c|}
\hline Methods & \multicolumn{2}{|c|}{$\begin{array}{l}\text { Features detected } \\
\text { in image pair }\end{array}$} & Inliers & $\begin{array}{l}\text { Matched } \\
\text { features }\end{array}$ & $\begin{array}{l}\text { Inlier } \\
\text { ratio }\end{array}$ & Methods & \multicolumn{2}{|c|}{$\begin{array}{l}\text { Features detected } \\
\text { in image pair }\end{array}$} & Inliers & $\begin{array}{l}\text { Matched } \\
\text { features }\end{array}$ & $\begin{array}{l}\text { Inlier } \\
\text { ratio }\end{array}$ \\
\hline ASIFT & 6485 & 6184 & 1236 & 1412 & 0.88 & SIFT & 431 & 444 & 117 & 143 & 0.82 \\
\hline ASURF & 13192 & 12398 & 1705 & 2351 & 0.73 & SURF & 832 & 843 & 123 & 162 & 0.76 \\
\hline AAKAZE & 2864 & 2586 & 840 & 1179 & 0.71 & AKAZE & 208 & 191 & 95 & 108 & 0.88 \\
\hline \multicolumn{12}{|c|}{ Image pair \#2 of A6 (S09, S06) } \\
\hline ASIFT & 6611 & 6184 & 191 & 365 & 0.52 & SIFT & 477 & 444 & 30 & 44 & 0.68 \\
\hline ASURF & 13411 & 12398 & 322 & 734 & 0.44 & SURF & 886 & 843 & 30 & 59 & 0.51 \\
\hline AAKAZE & 2530 & 2586 & 351 & 490 & 0.72 & AKAZE & 219 & 191 & 37 & 56 & 0.66 \\
\hline ABRISK & 4636 & 5164 & 203 & 296 & 0.69 & BRISK & 419 & 432 & 30 & 43 & 0.70 \\
\hline AORB & 12477 & 12917 & 335 & 555 & 0.60 & ORB & 500 & 500 & 32 & 52 & 0.62 \\
\hline ABRISK & 6476 & 5164 & 459 & 548 & 0.84 & BRISK & 643 & 432 & 70 & 74 & 0.95 \\
\hline AORB & 13486 & 12917 & 784 & 1027 & 0.76 & ORB & 500 & 500 & 61 & 75 & 0.81 \\
\hline \multicolumn{12}{|c|}{ Image pair \#4 of $A 6(S 11, S 06)$} \\
\hline ASIFT & 8556 & 6184 & 132 & 273 & 0.48 & SIFT & 581 & 444 & 30 & 40 & 0.75 \\
\hline ASURF & 13982 & 12398 & 195 & 502 & 0.39 & SURF & 931 & 843 & 35 & 53 & 0.66 \\
\hline AAKAZE & 3033 & 2586 & 200 & 299 & 0.67 & AKAZE & 271 & 191 & 31 & 47 & 0.66 \\
\hline ABRISK & 7262 & 5164 & 159 & 239 & 0.67 & BRISK & 720 & 432 & 32 & 42 & 0.76 \\
\hline AORB & 14158 & 12917 & 255 & 396 & 0.64 & ORB & 500 & 500 & 26 & 41 & 0.63 \\
\hline \multicolumn{12}{|c|}{ Image pair \#5 of $A 6(S 12, S 06)$} \\
\hline ASIFT & 9027 & 6184 & 426 & 624 & 0.68 & SIFT & 651 & 444 & 58 & 79 & 0.73 \\
\hline ASURF & 13988 & 12398 & 448 & 772 & 0.58 & SURF & 935 & 843 & 57 & 85 & 0.67 \\
\hline AAKAZE & 3031 & 2586 & 278 & 403 & 0.69 & AKAZE & 251 & 191 & 52 & 66 & 0.79 \\
\hline ABRISK & 7478 & 5164 & 365 & 452 & 0.81 & BRISK & 662 & 432 & 50 & 59 & 0.85 \\
\hline ASIFT & 4343 & 6184 & 80 & 140 & 0.57 & SIFT & 279 & 444 & 17 & 22 & 0.77 \\
\hline ASURF & 11170 & 12398 & 82 & 291 & 0.28 & SURF & 704 & 843 & 13 & 30 & 0.43 \\
\hline AAKAZE & 1885 & 2586 & 62 & 92 & 0.67 & AKAZE & 140 & 191 & 16 & 19 & 0.84 \\
\hline ABRISK & 3449 & 5164 & 43 & 57 & 0.75 & BRISK & 254 & 432 & 19 & 20 & 0.95 \\
\hline AORB & 10545 & 12917 & 76 & 143 & 0.53 & ORB & 500 & 500 & 21 & 34 & 0.62 \\
\hline \multicolumn{12}{|c|}{ Mean values for all image pairs } \\
\hline ASIFT & 6993 & 6184 & 418 & 564 & 0.74 & SIFT & 475.43 & 444 & 50.57 & 64.29 & 0.79 \\
\hline ASURF & 12916.29 & 12398 & 529.29 & 887.29 & 0.60 & SURF & 844.86 & 843 & 53.29 & 78.57 & 0.68 \\
\hline AAKAZE & 2657.71 & 2586 & 355.86 & 481.14 & 0.74 & AKAZE & 221.29 & 191 & 46.57 & 59 & 0.79 \\
\hline ABRISK & 5588.29 & 5164 & 320.86 & 391.86 & 0.82 & BRISK & 498 & 432 & 46.86 & 54.86 & 0.85 \\
\hline AORB & 12819.42 & 12917 & 559.57 & 755 & 0.74 & ORB & 500 & 500 & 50.29 & 66.14 & 0.76 \\
\hline
\end{tabular}

both areas. Thus, S17 was more blurred than other sessions for both areas. Figure $2 F, H$ displays the number of neurons common to each ROls Mask raw and $R O / s_{\text {Mask template }}^{\text {raw }}$ for the two areas. The results showed that S16 and S17 had smaller number of common neurons than other sessions of A5. S11 and S17 had smaller number of common neurons than other sessions of A6.

\section{Comparison between the fully affine invariant group and the feature-based group}

Tables 1, 2 show the quantitative comparison between the fully affine invariant group and the feature-based group of the A5 and A6, respectively. As can be seen, fully affine invariant methods generated more inliers than feature-based methods on sessions that had a low sharpness metrics or small common neuron number. For 


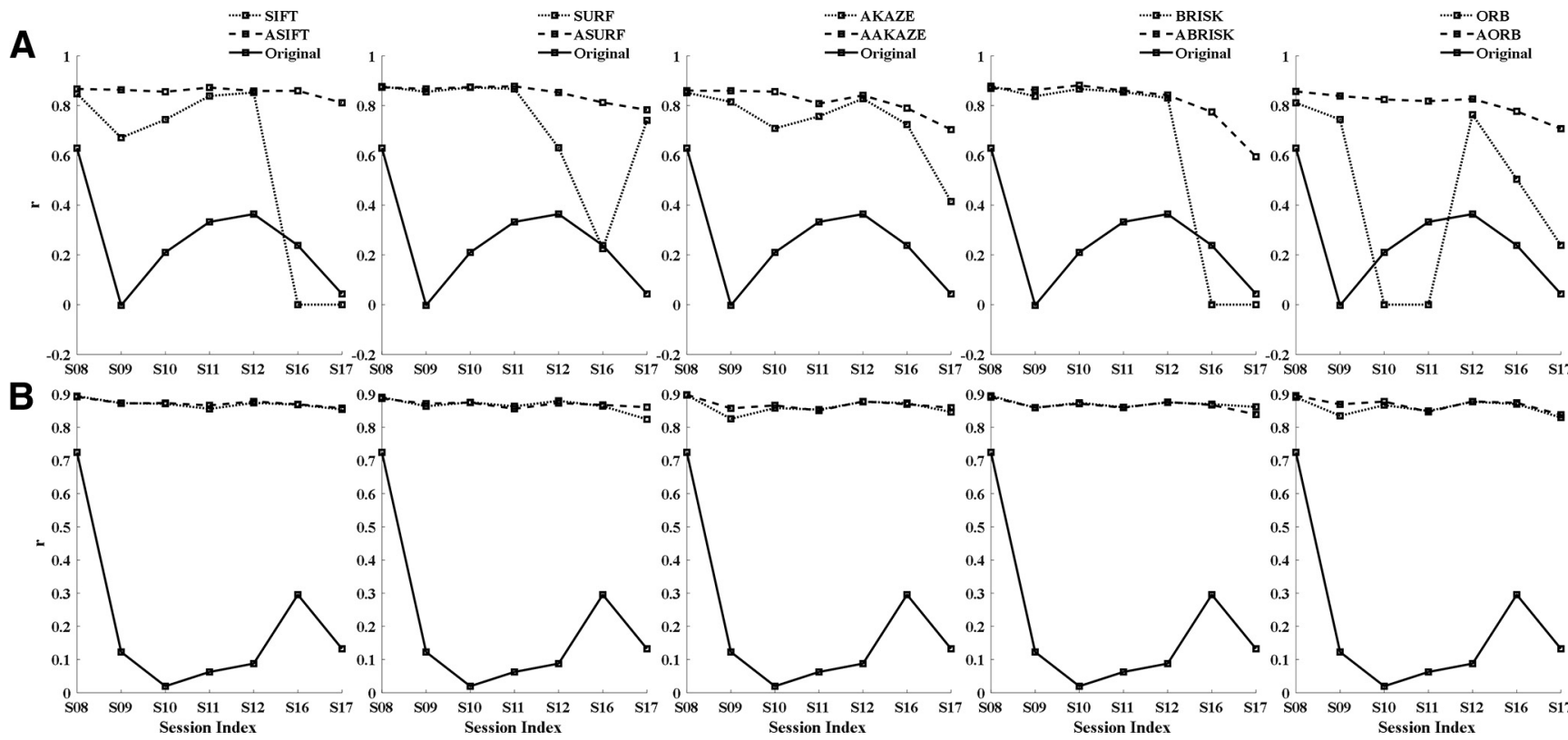

Figure 3. Comparison of performance between the fully affine invariant group and the feature-based group. The correlation between $R O / s_{\text {Mask template }}$ and the registered $R O$ ls $_{\text {Mask }}{ }_{n}^{\text {common }}, n \in(S 08, S 09, S 10, S 11, S 12, S 16, S 17)$ of different methods for area A5 $(\boldsymbol{A})$ and area A6 $(B)$. The results of the fully affine invariant group and the feature-based group are represented by dashed lines and dotted lines, respectively. The solid lines show the correlation coefficient of unregistered ROls mask-pair $\left\{R O / s_{\text {Mask }}{ }_{n}^{\text {common }}, R O l s_{\text {Mask }}^{\text {commonolate }}\right\}$.

instance, the inliers of ASIFT, ASURF, AAKAZE, ABRISK, and AORB were $20,20,13,12$, and 13 for $S 17$ of A5 while the inliers obtained by SIFT, SURF, AKAZE, BRISK, and ORB were $4,6,5,4$, and 7 for $S 17$ of $A 5$.

Figure 3 shows the correlation between ROls $s_{\text {Mask template }}$ and the registered $R O / s_{\text {Mask }}{ }_{\text {common }}$ for $\mathrm{A} 5$ and $\mathrm{A} 6 . n \in(\mathrm{S} 08$,
$S 09, S 10, S 11, S 12, S 16, S 17)$. Figure $3 A$ shows that fully affine invariant methods can reliably register FOV images across multiple sessions. In contrast, SIFT and BRISK failed to register the S16 and S17 of A5. Moreover, ORB failed to register the $S 10$ and $S 11$ of A5. Taken together, feature-based methods could easily fail when they cannot
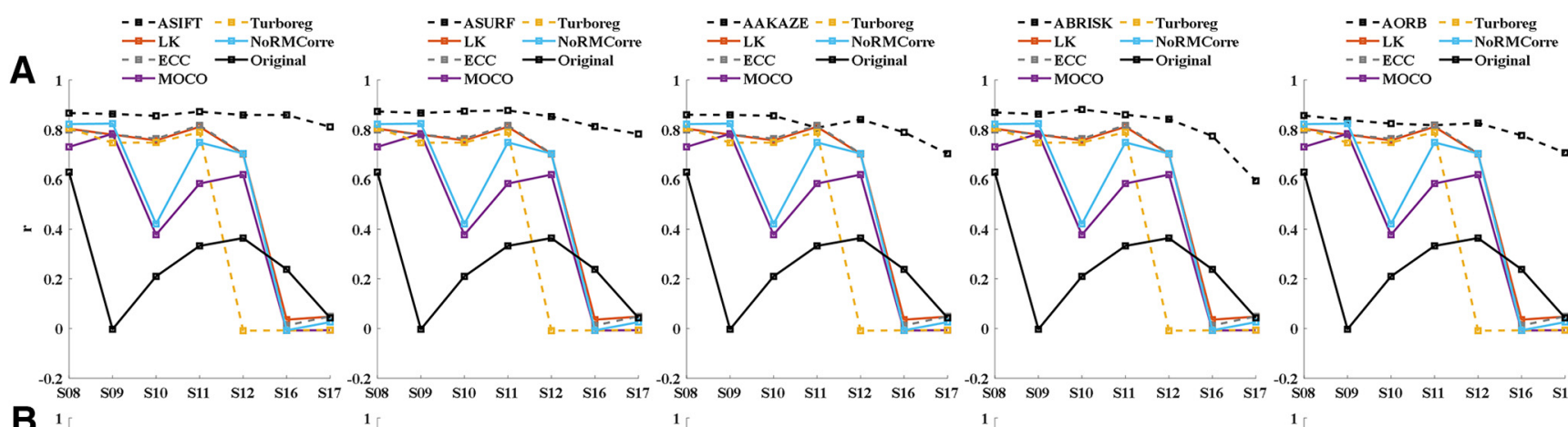

B
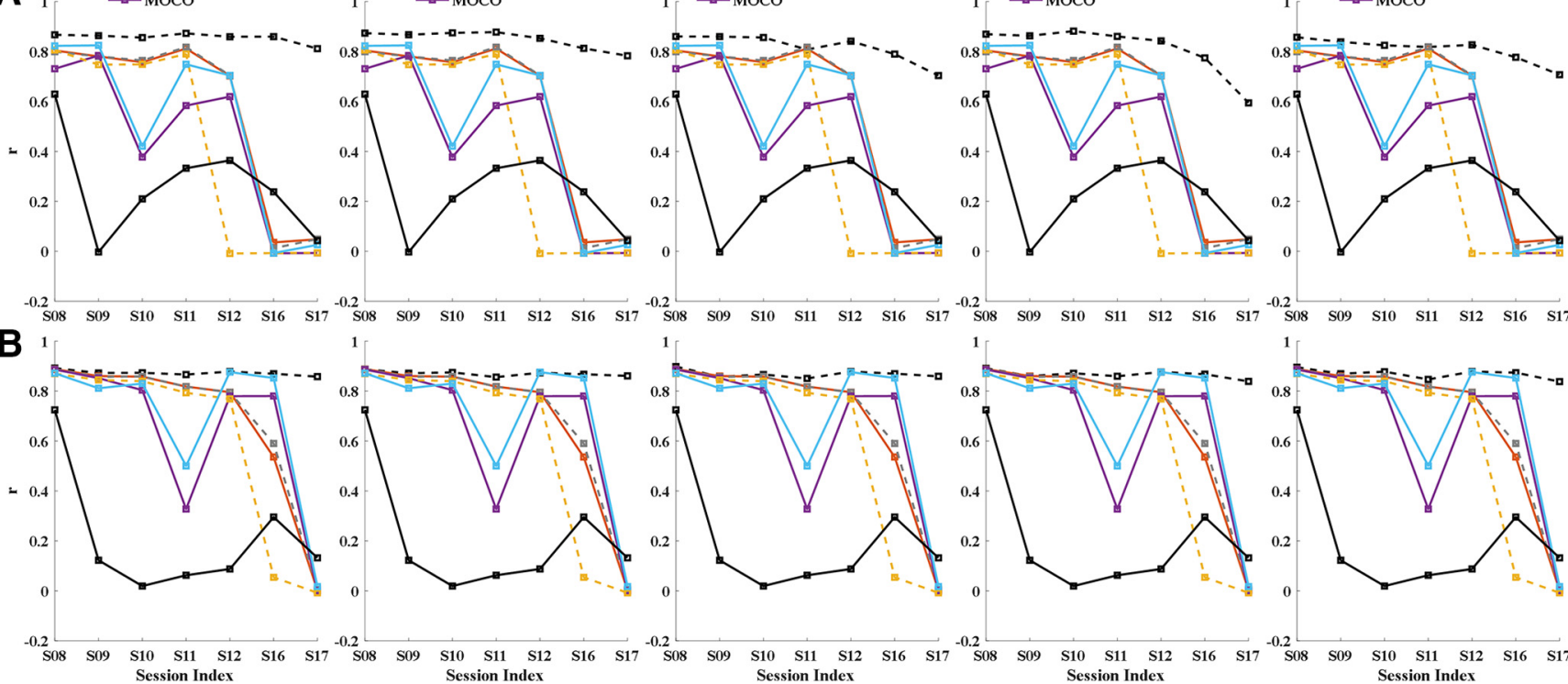

Figure 4. Comparison of performance between the fully affine invariant group and the conventional group. The correlation between $R O / s_{\text {Mask template }}^{\text {common }}$ and the registered ROIs Mask $_{n}^{\text {common }}, n \in(S 08, S 09, S 10, S 11, S 12, S 16, S 17)$ of different methods for area A5 $(\boldsymbol{A})$ and A6 (B). The correlation coefficients of the fully affine invariant group and the unregistered pairs of $\left\{R O / s_{\text {Mask }}{ }_{n}^{\text {common }}, R O / s_{\text {Mask }}\right.$ template $\}$ are represented by black dashed lines and black solid lines, respectively. The results of LK, ECC, MOCO, TurboReg, and NoRMCorre were shown in dark red, dashed gray, purple, dashed yellow, and blue color, respectively. 

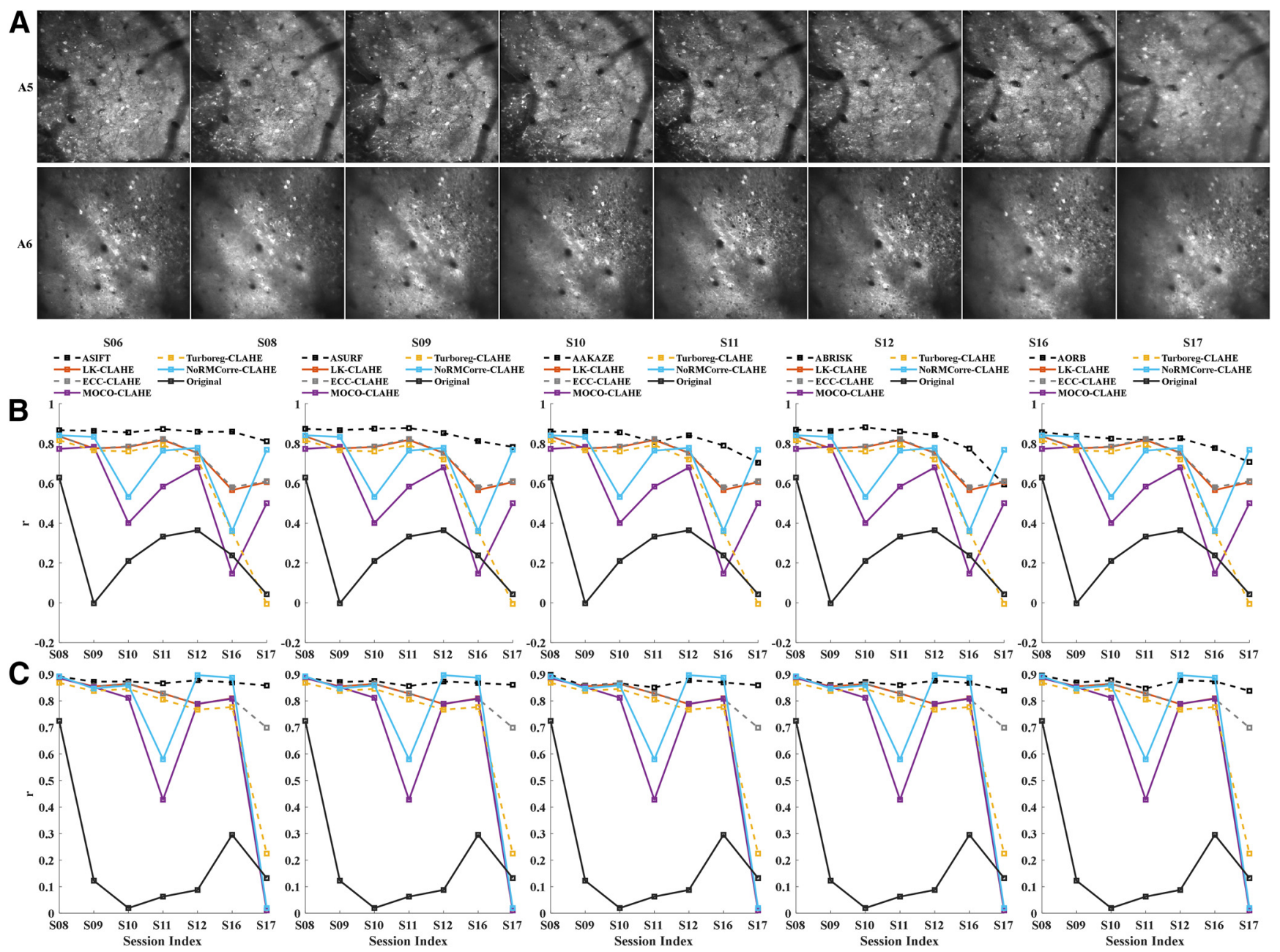

Figure 5. Comparison of performance between the fully affine invariant group and the CLAHE-based conventional group. A, The CLAHE adjusted FOV image of each session from A5 (upper row) and A6 (lower row). CLAHE, contrast limited adaptive histogram equalization. The correlation between $R O \mathrm{IS}_{\text {Mask }}$ common $_{\text {teme }}$ and the registered $R O \mathrm{Is}_{\text {Mask }}{ }_{n}^{\text {common }}, n \in(S 08, S 09, S 10, S 11, S 12, S 16, S 17)$ of different methods for area $\mathbf{A} 5(\boldsymbol{B})$ and $\mathrm{A} 6(\boldsymbol{C})$. The correlation coefficients of the fully affine invariant group and the unregistered pairs of $\left\{R O I s_{\text {Mask }}{ }_{n}^{\text {common }}, R O I s_{\text {Mask }}\right.$ temmolate $\}$ are represented by black dashed lines and black solid lines, respectively. The results of LKCLAHE, ECC-CLAHE, MOCO-CLAHE, TurboReg-CLAHE, and NoRMCorre-CLAHE were shown in dark red, dashed gray, purple, dashed yellow, and blue color, respectively.

generate enough inliers. For area A6, the fully affine invariant group and feature-based group achieved similar results (Fig. 3B). In addition, Tables 1, 2 show that the mean ratios of inliers to initial matches (Inliers/Matched features) of the fully affine invariant group is lower than that of the feature-based group for both areas.

\section{Comparison between the fully affine invariant group and the conventional group}

Different parameters were tested for methods in the conventional group to maximize their performance. The iteration of LK and ECC was set to 100 , the number of levels for multiresolution was set to 3 , and a total of four different transformation types (affine, translation, Euclidean, and homography) were compared on all FOV pairs. After comparison, Euclidean was applied because it produced the best results. For the non-rigid NoRMCorre method, five different square patch sizes $(24,32,48,96,128)$ were tested with other parameters set as default values. Finally, the default patch size value 32 was adopted in the current study. For TurboReg, four different transformation types (affine, translation, rigid body, and bilinear) were examined on all FOV pairs. Lastly, a rigid body was employed in this study. For MOCO, the default parameters were used.

In Figure 4, we compared each method in the fully affine invariant group with all methods in the conventional group. As can be seen, ASIFT, ASURF, AAKAZE, ABRISK, and AORB outperformed the methods in the conventional group for most sessions from A5 and A6. For A5, all methods in the conventional group failed to register sessions that had low sharpness metrics (S17) or few common neurons (S16; Fig. 4A). For A6, the intensity-based methods, i.e., LK, ECC, and TurboReg, failed when the session had both a low sharpness 


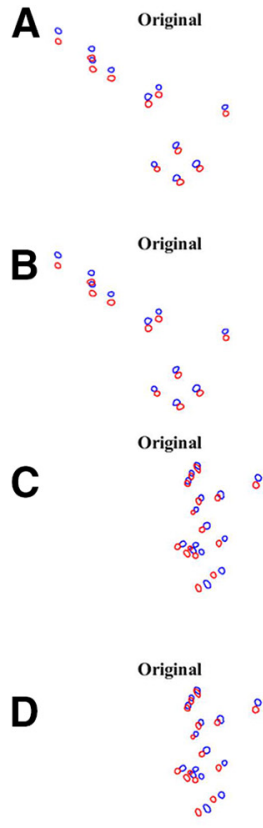

S17
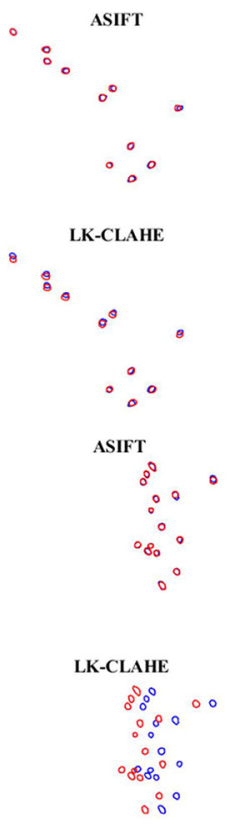

S17
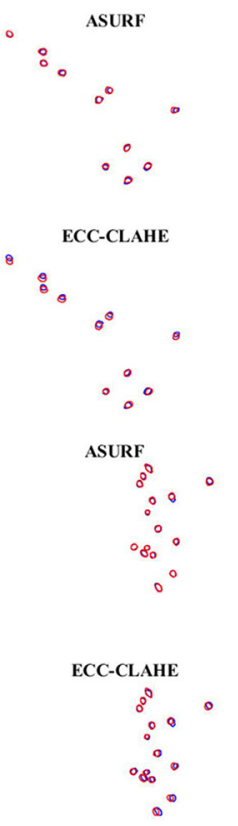

S17
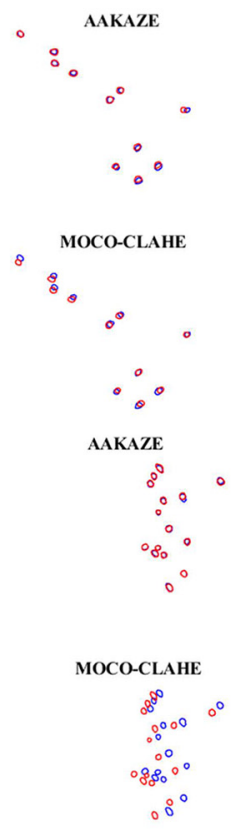

S17
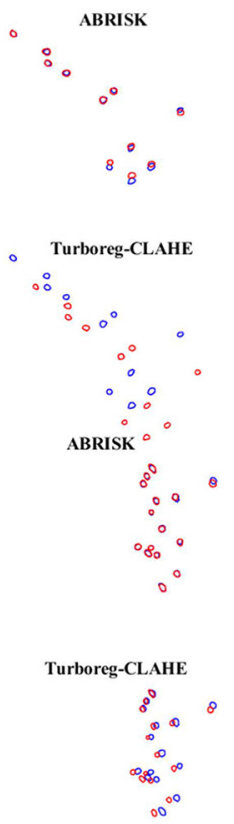

S17

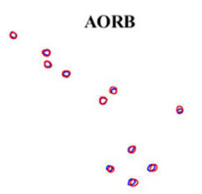

- NoR
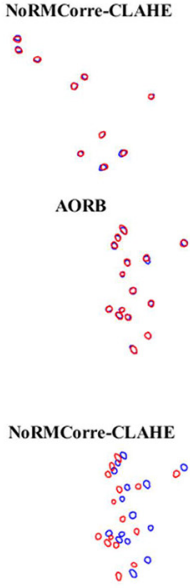

S17

Figure 6. Visualization of the overlay of the ROls mask-pairs on $\mathrm{S} 17$ of $\mathrm{A} 5$ and $\mathrm{A} 6$. The overlay of the RO/s Mask template (blue) and the registered $R O / s_{\text {Mask }}{ }_{n}^{\text {common }}$ (red) on S17 of the fully affine invariant group $(\boldsymbol{A})$ and the CLAHE-based conventional group (B) for area A5. The overlay of the ROIs Mask template $_{\text {common }}$ (blue and the registered ROIs Mask $_{n}^{\text {common }}$ (red) on $\mathrm{S} 17$ of the fully affine invariant group $(\boldsymbol{C})$ and the CLAHE-based conventional group $(\boldsymbol{D})$ for area A6. The higher degree of overlap between the template and the registered ROls mask, the better the alignment results.

metrics and a small common neuron number (S17). Moreover, the performance of $\mathrm{MOCO}$ and non-rigid NoRMCorre decreased in sessions with fewer common neurons (S11 and S17; Fig. 4B).

\section{Comparison between the fully affine invariant group and the CLAHE-based conventional group}

We tested different parameters for CLAHE, and finally a block size of $8 \times 8$ and contrast limiting threshold $=1$ were adopted in this study. Figure $5 A$ shows the CLAHE adjusted FOV image of each session from A5 and A6. As can be seen, the local details in the images are more recognizable when compared with the results shown in Figure 2A,C.

Fully affine invariant methods were compared with CLAHE-based conventional methods (Fig. 5B,C). Results showed that ASIFT, ASURF, AAKAZE, ABRISK, and AORB outperformed the methods in the CLAHE-based conventional group for most of sessions from both $\mathrm{A} 5$ and A6. The performance of MOCO-CLAHE and non-rigid NoRMCorre-CLAHE decreased in sessions with small common neuron number (S16 for A5; S11 and S17 for A6). Besides, TurboReg-CLAHE failed to register the low sharpness session S17 for A5 and A6. Figure 6 visualizes the alignment results on $\mathrm{S} 17$ of the fully affine invariant group and the CLAHE-based conventional group for A5 (Fig. $6 A, B$ ) and $A 6$ (Fig. $6 C, D$ ), respectively. The higher degree of overlap between the template and the registered ROls mask, the better the alignment results. The overlap results are in line with the results shown in Figure 5B,C.

\section{The mean and standard error of alignment results for the four groups}

The mean \pm SEM of the correlation on all registered

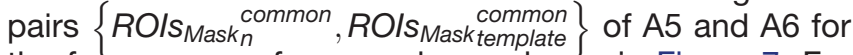
the four groups of approach are shown in Figure 7. For A5, the results indicate that the fully affine invariant group achieves better results than all other groups. Moreover, the fully affine invariant group outperforms the conventional group and the CLAHE-based conventional group for A6. Besides, methods in the CLAHE-based conventional group outperform the corresponding methods in conventional group for both areas.

\section{Discussion}

In this study, we introduce new methodologies for cross-session FOV alignment. We explore the performance of ASIFT, ASURF, AAKAZE, ABRISK, and AORB on FOV alignment of in vivo calcium imaging data, and we improve their reliability by using L1-norm. Furthermore, we compare their performance with general feature-based methods (SIFT, SURF, AKAZE, BRISK, ORB), the conventional methods (LK, ECC, MOCO, TurboReg, NoRMCorre), and the CLAHE-based conventional methods (LK-CLAHE, ECCCLAHE, MOCO-CLAHE, TurboReg-CLAHE, NoRMCorreCLAHE). Our results show that the fully affine invariant methods outperform the other methods in general and also in the case of image blurring, insufficient common neurons, and uneven background brightness. To the best of our knowledge, this is the first study that proves the feasibility of fully affine invariant methods in cross-session calcium FOV alignment. 

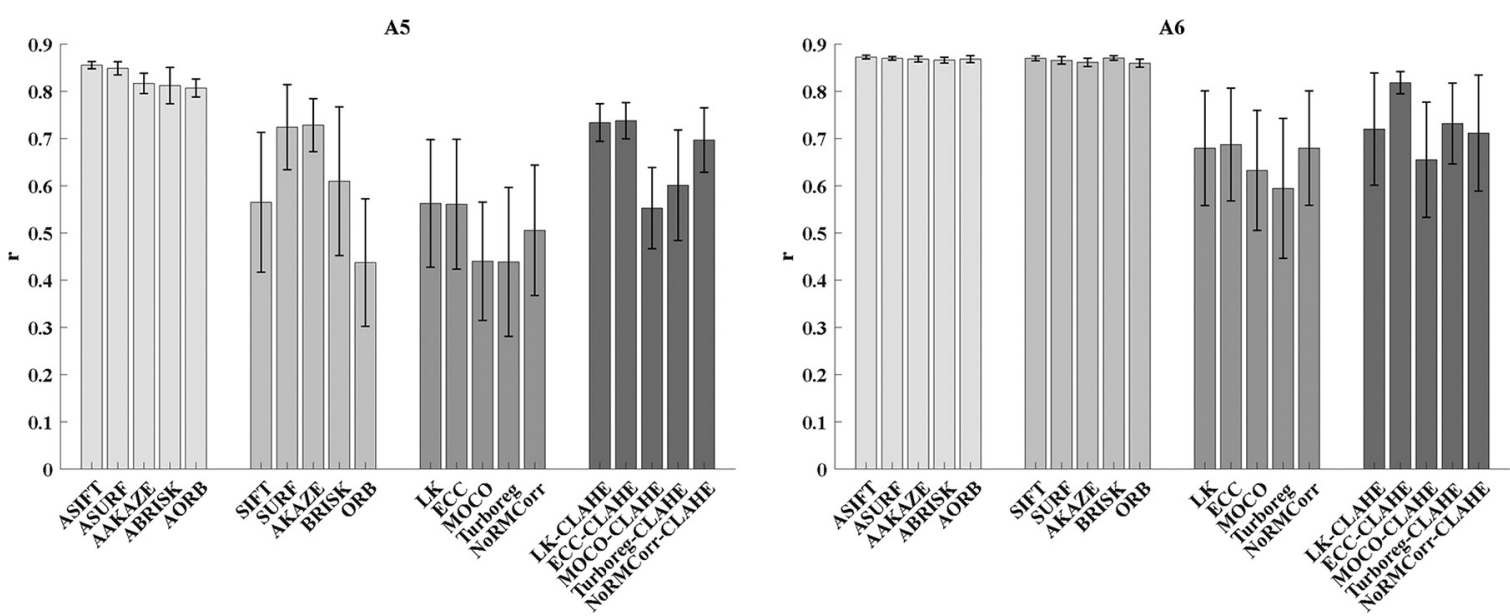

Figure 7. The mean $\pm \mathrm{SEM}$ of the correlations on all registered ROls mask-pairs $\left\{R O / s_{\text {Mask }}{ }_{n}^{\text {common }}, R O / s_{\text {Mask }}^{\text {common }}\right.$ template $\}$ of area A5 (left) and $A 6$ (right) for the four groups of approach.

These methods could be useful for neuroscience research, especially for studies involving experience-dependent plasticity spanning over days or weeks.

Fully affine invariant methods outperform feature-based methods because they use different ways to extract keypoints. Specifically, feature-based methods only detect keypoints on original image pair, while fully affine-invariant methods also detect keypoints on simulated images caused by change of the viewing angle. However, the potential drawback is that full affine-invariant methods are more prone to accumulation of keypoints that are not discriminative. As a result, it could be difficult for NNDR and RANSAC to match keypoints as well as to keep inliers when the discrimination is low. Studies have shown that when the ratio of inliers to initial matches is low, methods like RANSAC are unlikely to find a good solution since it does not test enough hypotheses (Raguram et al., 2008). Moreover, RANSAC could produce different results each time because of its randomness (Hast et al., 2013). We tried to solve this problem by replacing RANSAC with other advanced methods, i.e., progressive sample consensus (PROSAC; Chum and Matas, 2005) and grid-based motion statistics (Bian et al., 2017). However, both methods cannot generate reproducible results when the ratio of inliers to initial matches is low (data not shown). In this study, we overcome this problem by repetitively running the NNDR and RANSAC for multiple times (100 times in the current study) and choosing the transformation matrix which minimizes L1-norm of the intensity difference where neuron appears between $\left\{R O I s_{\text {Maskn }}^{\text {raw }}, R O I s_{\text {Mask template }}\right\}$. After using this simple operation, fully affine invariant methods can achieve reproducible results even if they have a low inlier ratio.

Methods in the conventional group decrease their performance in sessions with small common neuron number for various reasons. Intensity-based methods, i.e., LK, ECC, and TurboReg, register images based on global intensity information. When the common neuron number is small, the intensity difference of the non-common area may have a larger impact on the results of registration than that of the common area, making it difficult to find the optimal solution. MOCO and non-rigid NoRMCorre register the image pair using frequency domain information within the whole image or single patch. MOCO cannot correct rotation artifacts which frequently happen in cross-session imaging. Non-rigid NoRMCorre may not be applicable when the patch does not contain enough spatial features (Mitani and Komiyama, 2018). In contrast, the fully affine invariant group registers the image pair using local keypoints as a statistic of the image content. They avoid to use global image content, thereby decreasing the negative effects of the different contents in the FOV image pair. Therefore, the fully affine invariant group outperforms the conventional group in the case of insufficient common neurons. Additionally, CLAHE increases the accuracy of methods in the conventional group because it improves image characteristics of uneven brightness regions. CLAHE enhances local image details by directly manipulating the intensity values of individual pixels in each image block. However, the results of methods in CLAHEbased conventional group are still inferior to those of fully affine invariant methods in most sessions. In other words, fully affine invariant methods do not require CLAHE to obtain reliable results. Thus, fully affine invariant methods are robust to uneven background brightness.

In this study, we improve the reliability of the fully affine invariant group by using an L1-norm. However, an alternative could be to use dimension reduction methods, such as principal components analysis, to increase the discrimination of keypoints. Besides, here, fully affine invariant methods are applied as offline methods for FOV alignment. It would be desirable to extend them as online registration methods, which will help the experimenter to more efficiently collect the same group of neurons across days or weeks in the experiments. Moreover, we did not include SIMA (Kaifosh et al., 2014) and Suite2p (Pachitariu et al., 2017) in our study, because alignment methods adopted by SIMA and Suite2p are not designed for multiday recording. However, we compared our 
proposed methods with the built-in method of CalmAn, i.e., NoRMCorre.

This study is the first and comprehensive work investigating the performance of ASIFT, ASURF, AAKAZE, ABRISK, and AORB on longitudinal cellular resolution calcium imaging data. These methods will be useful for many neuroscience studies involving chronic changes in neuronal activities. Moreover, although ASIFT, ASURF, AAKAZE, ABRISK, and AORB are conducted on twophoton microscopy-based calcium imaging data, these methods should be promising in registering FOV images collected by one-photon or widefield fluorescence microscopy.

\section{References}

Alcantarilla PF, Solutions T (2011) Fast explicit diffusion for accelerated features in nonlinear scale spaces. IEEE Trans Patt Anal Mach Intell 34:1281-1298.

Baker S, Matthews I (2004) Lucas-Kanade 20 years on: a unifying framework. Int J Comput Vision 56:221-255.

Bay H, Ess A, Tuytelaars T, Van Gool L (2008) Speeded-up robust features (SURF). Comput Vision Image Underst 110:346-359.

Bian J, Lin WY, Matsushita Y, Yeung SK, Nguyen TD, Cheng MM (2017) GMS: grid-based motion statistics for fast, ultra-robust feature correspondence. Proceedings of the IEEE Conference on Computer Vision and Pattern Recognition, pp 4181-4190, Honolulu, Hawaii.

Bradski G (2000) The OpenCV library. Dr Dobb's Journal of Software Tools 25:120-125.

Chen H, He Y, Wei L, Li X, Zhang Y (2019) Automatic dewarping of retina images in adaptive optics confocal scanning laser ophthalmoscope. IEEE Access 7:59585-59599.

Chum O, Matas J (2005) Matching with PROSAC-progressive sample consensus. 2005 IEEE Computer Society Conference on Computer Vision and Pattern Recognition, pp 220-226, San Diego, $\mathrm{CA}$

De K, Masilamani V (2013) Image sharpness measure for blurred images in frequency domain. Procedia Eng 64:149-158.

Dubbs A, Guevara J, Yuste R (2016) moco: fast motion correction for calcium imaging. Front Neuroinform 10:6.

Evangelidis G (2013) IAT: a MATLAB toolbox for image alignment. Natick: MathWorks.

Evangelidis G, Psarakis E (2008) Parametric image alignment using enhanced correlation coefficient maximization. IEEE Trans Pattern Anal Mach Intell 30:1858-1865.

Fischler M, Bolles R (1981) Random sample consensus: a paradigm for model fitting with applications to image analysis and automated cartography. Commun ACM 24:381-395.

Giovannucci A, Friedrich J, Gunn P, Kalfon J, Brown BL, Koay SA, Khakh BS, Taxidis J, Najafi F, Gauthier JL, Zhou P, Khakh BS, Tank DW, Chklovskii DB, Pnevmatikakis EA (2019) CalmAn an open source tool for scalable calcium imaging data analysis. Elife 8:e38173.

Grewe B, Gründemann J, Kitch L, Lecoq J, Parker J, Marshall J, Larkin M, Jercog P, Grenier F, Li J, Lüthi A, Schnitzer MJ (2017) Neural ensemble dynamics underlying a long-term associative memory. Nature 543:670-675.

Han S, Yang W, Yuste R (2019) Two-color volumetric imaging of neuronal activity of cortical columns. Cell Rep 27:2229-2240.e4.

Hast A, Nysjö J, Marchetti A (2013) Optimal ransac-towards a repeatable algorithm for finding the optimal set. J WSCG 21:21-30.

Heo C, Park H, Kim Y, Baeg E, Kim Y, Kim S, Suh M (2016) A soft, transparent, freely accessible cranial window for chronic imaging and electrophysiology. Sci Rep 6:27818.
Hwang E, Link T, Hu Y, Lu S, Wang E, Lilascharoen V, Aronson S, Neil K, Lim B, Komiyama T (2019) Corticostriatal flow of action selection bias. Neuron 104:1126-1140.e6.

Kaifosh P, Zaremba J, Danielson N, Losonczy A (2014) SIMA: Python software for analysis of dynamic fluorescence imaging data. Front Neuroinform 8:80.

Leutenegger S, Chli M, Siegwart R (2011) BRISK: binary robust invariant scalable keypoints. 2011 IEEE International Conference on Computer Vision, pp 2548-2555.

Lichtman JW, Conchello JA (2005) Fluorescence microscopy. Nat Methods 2:910-919.

Lowe D (2004) Distinctive image features from scale-invariant keypoints. Int J Comput Vision 60:91-110.

Mitani A, Komiyama T (2018) Real-time processing of two-photon calcium imaging data including lateral motion artifact correction. Front Neuroinform 12:98.

Namboodiri VMK, Otis JM, van Heeswijk K, Voets ES, Alghorazi RA, Rodriguez-Romaguera J, Mihalas S, Stuber GD (2019) Single-cell activity tracking reveals that orbitofrontal neurons acquire and maintain a long-term memory to guide behavioral adaptation. Nat Neurosci 22:1110-1121

Pachitariu M, Stringer C, Schröder S, Dipoppa M, Rossi LF, Carandini M, Harris KD (2017) Suite2p: beyond 10,000 neurons with standard two-photon microscopy. bioRxiv. doi: https://doi. org/10.1101/061507.

Pakan J, Francioni V, Rochefort N (2018) Action and learning shape the activity of neuronal circuits in the visual cortex. Curr Opin Neurobiol 52:88-97.

Pnevmatikakis E, Giovannucci A (2017) NoRMCorre: an online algorithm for piecewise rigid motion correction of calcium imaging data. J Neurosci Methods 291:83-94.

Raguram R, Frahm JM, Pollefeys M (2008) A comparative analysis of RANSAC techniques leading to adaptive real-time random sample consensus, pp 500-513. Marseille: European Conference on Computer Vision.

Reza A (2004) Realization of the contrast limited adaptive histogram equalization (clahe) for real-time image enhancement. J VLSI Signal Proc Syst Signal Image Video Technol 38:35-44.

Rublee E, Rabaud V, Konolige K, Bradski G (2011) ORB: an efficient alternative to SIFT or SURF. ICCV 11:2.

Sheintuch L, Rubin A, Brande-Eilat N, Geva N, Sadeh N, Pinchasof O, Ziv Y (2017) Tracking the same neurons across multiple days in ca2+ imaging data. Cell Reports 21:1102-1115.

Stanciu S, Hristu R, Boriga R, Stanciu G (2010) On the suitability of sift technique to deal with image modifications specific to confocal scanning laser microscopy. Microsc Microanal 16:515-530.

Stringer C, Pachitariu M, Steinmetz N, Carandini M, Harris K (2019) High-dimensional geometry of population responses in visual cortex. Nature 571:361-365.

Stringer C, Michaelos M, Pachitariu M (2020) Cellpose: a generalist algorithm for cellular segmentation. bioRxiv. doi: https://doi.org/ 10.1101/2020.02.02.931238.

Thévenaz P, Ruttimann U, Unser M (1998) A pyramid approach to subpixel registration based on intensity. IEEE Trans Image Process 7:27-41.

Ûnay D, Stanciu S (2018) An evaluation on the robustness of five popular keypoint descriptors to image modifications specific to laser scanning microscopy. IEEE Access 6:40154-40164.

Wagner MJ, Kim TH, Kadmon J, Nguyen ND, Ganguli S, Schnitzer MJ, Luo L (2019) Shared cortex-cerebellum dynamics in the execution and learning of a motor task. Cell 177:669-682.e24.

Yu G, Morel J (2011) ASIFT: an algorithm for fully affine invariant comparison. Image Proc On Line 1:11-38.

Yu G, Morel J (2009) A fully affine invariant image comparison method. 2009 IEEE International Conference on Acoustics, Speech and Signal Processing, pp 1597-1600, Taipei, Taiwan. 